\title{
Optimisation of oil red $O$ staining permits combination with immunofluorescence and automated quantification of lipids
}

Citation for published version (APA):

Koopman, R., Schaart, G., \& Hesselink, M. K. C. (2001). Optimisation of oil red O staining permits combination with immunofluorescence and automated quantification of lipids. Histochemistry and Cell Biology, 116(1), 63-68. https://doi.org/10.1007/S004180100297

Document status and date:

Published: 01/01/2001

DOI:

10.1007/S004180100297

Document Version:

Publisher's PDF, also known as Version of record

Document license:

Taverne

Please check the document version of this publication:

- A submitted manuscript is the version of the article upon submission and before peer-review. There can be important differences between the submitted version and the official published version of record.

People interested in the research are advised to contact the author for the final version of the publication, or visit the DOI to the publisher's website.

- The final author version and the galley proof are versions of the publication after peer review.

- The final published version features the final layout of the paper including the volume, issue and page numbers.

Link to publication

\footnotetext{
General rights rights.

- You may freely distribute the URL identifying the publication in the public portal. please follow below link for the End User Agreement:

www.umlib.nl/taverne-license

Take down policy

If you believe that this document breaches copyright please contact us at:

repository@maastrichtuniversity.nl

providing details and we will investigate your claim.
}

Copyright and moral rights for the publications made accessible in the public portal are retained by the authors and/or other copyright owners and it is a condition of accessing publications that users recognise and abide by the legal requirements associated with these

- Users may download and print one copy of any publication from the public portal for the purpose of private study or research.

- You may not further distribute the material or use it for any profit-making activity or commercial gain

If the publication is distributed under the terms of Article $25 \mathrm{fa}$ of the Dutch Copyright Act, indicated by the "Taverne" license above, 
René Koopman · Gert Schaart

Matthijs K.C. Hesselink

\section{Optimisation of oil red 0 staining permits combination with immunofluorescence and automated quantification of lipids}

\begin{abstract}
The objective of the present study was to develop a stain permitting automated quantification of myocellular lipid depositions in skeletal muscle sections together with immunolocalisation of other myocellular constituents by fluorescence microscopy. Lipid droplets were detected in skeletal muscle by oil red O (ORO). Conventional ORO was modified to diminish background staining, prevent crystallisation of ORO and to optimise lipid retention in cryosections. These modifications resulted in a punctate staining of lipid droplets, rather than the somewhat diffuse staining by conventional ORO. Small cavities in muscle sections (like the lumen of small blood vessels) lack ORO when using the protocol presented here. In addition a staining protocol is presented combining ORO with immunofluorescence. This combination permits multiple staining studies in the same section. Thus, lipid droplets can be studied together with immunolabelling of proteins involved in lipid handling and metabolism. This will extend our knowledge on the subcellular localisation of lipid handling proteins (i.e. enzymes and fatty acid transporting proteins) in relation to the localisation of lipid depositions. In conclusion, the protocol presented here permits examination of ORO-stained lipid droplets in skeletal muscle sections together with multiple staining of other immunodetectable proteins present in skeletal muscle by quantitative fluorescence microscopy.
\end{abstract}

Keywords Lipid droplets - Skeletal muscle . Fluorescence microscopy $\cdot$ Multiple staining

R. Koopman · G. Schaart · M.K.C. Hesselink ( $)$

Department of Movement Sciences,

Nutrition and Toxicology Research Institute Maastricht (NUTRIM),

Maastricht University, PO Box 616, 6200 MD, Maastricht,

The Netherlands

e-mail: matthijs.Hesselink@BW.unimaas.nl

Tel.: +31-43-3881317, Fax: +31-43-3670972

\section{Introduction}

Dietary fats can be oxidised and stored in skeletal muscle cells (Vusse and Reneman 1996). The myocellular storage of lipids has regained scientific interest in research towards obesity and type 2 diabetes since it has been shown that intramyocellular triglycerides (lipid droplets or IMTG) are linked with insulin resistance (Goodpaster et al. 2000a; Krssak et al. 1999; Pan et al. 1997; Russell et al. 1998). IMTG and its oxidation has been examined by tracer techniques, permitting the differentiation between plasma and non-plasma derived fatty acid oxidation (Guo and Jensen 1998; Schrauwen et al. 2000), or by chemical extraction of the lipids from muscle biopsies (Dyck and Bonen 1998; Dyck et al. 1997). Also microdissection of single muscle fibres, either chemically or mechanically, can be used to examine intramyocellular substrate metabolism (Pette et al. 1999). However, we are not aware of papers quantitatively examining lipid deposition in single fibres, possibly due to loss of lipid in the permeabilisation procedure of the fibres. Nowadays, IMTG can also be quantified non-invasively using proton magnetic resonance spectroscopy (Boesch et al. 1997). Visualisation of IMTG can be performed by classic histochemical stains like oil red O, Sudan black and osmium tetroxide (Bayliss-High 1977). In contrast to conventional histochemical stains none of the other approaches to examine lipid depositions in skeletal muscle permit studies towards the precise subcellular localisation of IMTG, towards the potential heterogeneity between different muscle fibres or towards colocalisation of IMTG with enzymes which play a crucial role in transport, deposition, mobilisation and oxidation of fatty acids.

Using oil red $\mathrm{O}$, neutral lipids (mainly triglycerides) are stained with an orange-red tint (Lillie and Ashburn 1943). Different groups have used this method successfully to demonstrate IMTG (Goodpaster et al. 2000b; Russell et al. 1998). Goodpaster et al. (2000b), using an optimised oil red $\mathrm{O}$ staining protocol combined with computerised greyscale analysis, elegantly showed that 
the number of lipid droplets as well as the area of IMTG was increased in obese and type 2 diabetic subjects. Moreover, they showed in replication studies that the number and area of the lipid droplets was not affected when the stains were performed on the same sections on different occasions by different people (Goodpaster et al. $2000 \mathrm{~b}$ ). This study has provided a sound foundation to use oil red $\mathrm{O}$ to quantitatively study IMTG.

Combining conventional oil red $\mathrm{O}$ with other (immuno)histochemical stains may provide the clue to many of the currently prevailing questions on the exact subcellular localisation of IMTG and its potential vicinity with proteins involved in lipid metabolism. The combined use of oil red $\mathrm{O}$ with classic immunohistochemical stains developed for bright-field microscopy, like 3,3'-diaminobenzidin hydrochloride or 3-amino-9-ethylcarbazole, will raise stains of alike colour. When captured images of these stains are converted to greyscale for computerised quantification it will be complex to differentiate between the different stains. In addition, these classic stains raise a signal which is less clear and sharp than can be achieved by fluorescence microscopy, which may complicate the precise cellular localisation.

To perform automated quantification of lipids in muscle sections, together with examination of other immunolabelled subcellular constituents, we aimed to optimise oil red $\mathrm{O}$ for use in quantitative fluorescence microscopy.

\section{Materials and methods}

\section{Muscle samples}

Skeletal muscle samples were obtained from mature Wistar rats (aged 12-15 weeks) and human volunteers. Muscles characterised by a high oxidative capacity (soleus) and a high glycolytic capacity (extensor digitorum longus) were obtained from rats, while human $\mathrm{m}$. vastus lateralis biopsies were examined.

Muscles were dissected carefully, freed from any visible fat and blood, rapidly frozen in liquid nitrogen-cooled isopentane and embedded in Tissue-Tek (Sakura Finetek Europe, Zoeterwoude, The Netherlands). Serial sections $(4 \mu \mathrm{m})$ were thaw mounted on uncoated precleaned $(96 \%$ ethanol) glass slides. Before processing, or storage at $-70^{\circ} \mathrm{C}$, the samples were air dried for $15 \mathrm{~min}$.

\section{Chemicals}

A $3.7 \%$ formaldehyde solution was made by diluting the $37 \%$ stock solution (Merck, Darmstadt, Germany) with deionised water. Oil red O was obtained from Fluka Chemie (C.I. number 26125; Buchs, Switzerland) and dissolved to a stock solution by adding $500 \mathrm{mg}$ oil red $\mathrm{O}$ to $100 \mathrm{ml} 60 \%$ triethyl-phosphate $\left[\left(\mathrm{C}_{2} \mathrm{H}_{5} \mathrm{O}\right)_{3} \mathrm{PO}\right.$; Fluka Chemie]. Prior to staining, a $36 \%$ triethyl phosphate working solution, containing $12 \mathrm{ml}$ oil red $\mathrm{O}$ stock solution and $8 \mathrm{ml}$ deionised water, was prepared. This solution was filtered through Whatman paper number 42 (Whatman, Maidstone, UK) to remove crystallised oil red $\mathrm{O}$.

As a buffer, phosphate-buffered saline [PBS: $137 \mathrm{mM}$ sodium chloride ( $\mathrm{NaCl}$; Merck), $3 \mathrm{mM}$ potassium chloride ( $\mathrm{KCl}$; Merck), $8 \mathrm{mM}$ disodium hydrogen phosphate $\left(\mathrm{Na}_{2} \mathrm{HPO}_{4}\right.$; Merck), $3 \mathrm{mM}$ potassium dihydrogen phosphate $\left(\mathrm{KH}_{2} \mathrm{PO}_{4}\right.$; Merck), $\mathrm{pH}$ 7.4] was used for dilution of Triton $\mathrm{X}-100$, glycerol and antibodies.
Haematoxylin solution according to Mayer (Fluka Chemie) was used to visualise nuclei and a $10 \%$ glycerol (Fluka Chemie) solution in PBS was used as an embedding medium for oil red O-stained sections.

In the procedure for immunofluorescence $0.5 \%$ Triton X-100 (Merck) in PBS was prepared. Primary and secondary antibodies were appropriately diluted either in PBS or in $0.1 \%$ Tween 20 (Sigma, St. Louis, Mo., USA) in PBS. As an embedding medium for immunofluorescence a $10 \%$ glycerol (Fluka Chemie) in $10 \mathrm{mM}$ TRIS-HCl (Merck), $\mathrm{pH} 8.5$, containing $0.1 \mu \mathrm{g} / \mathrm{ml} \mathrm{4}$ 4 $^{\prime}, 6-$ diamidine-2'-phenylindole dihydrochloride (DAPI; Roche Diagnostics Nederland, Almere, The Netherlands) was used.

\section{Oil red O staining}

Three different oil red $\mathrm{O}$ staining protocols were used in the present study. Initially, conventional oil red $\mathrm{O}$ staining (Lillie and Ashburn 1943) was performed. As we apparently lost the majority of the stained lipids in the differentiation and rinsing steps performed after the oil red $\mathrm{O}$ stain, we successively performed the procedure described by Goodpaster et al. (2000b), where the differentiation procedure is skipped. Using this procedure lipids were stained, albeit somewhat diffusely, and a few oil red $\mathrm{O}$ crystals remain (discussed in detail in Results section).

In order to overcome crystallisation and to realise a less diffuse staining, we modified the conventional staining protocol as follows. We performed chemical fixation $(1 \mathrm{~h})$ of the air-dried, thawed, sections using $3.7 \%$ formaldehyde solution in deionised water. Moreover, we diluted oil red $\mathrm{O}$ in triethyl phosphate $\left[\left(\mathrm{C}_{2} \mathrm{H}_{5} \mathrm{O}\right)_{3} \mathrm{PO}\right]$ instead of isopropanol $\left[\left(\mathrm{CH}_{3}\right)_{2} \mathrm{CHOH}\right]$. Accordingly, the staining protocol started with $1 \mathrm{~h}$ fixation by $3.7 \%$ formaldehyde. Excess formaldehyde was removed by three rinses in deionised water for $30 \mathrm{~s}$. Subsequently, the sections were immersed in the working solution of oil red $\mathrm{O}$ for $30 \mathrm{~min}$. Hereafter, sections were washed with three exchanges of deionised water for 3 times 30 s. If desired, sections were counterstained using Mayer's haematoxylin for $60 \mathrm{~s}$ to visualise nuclei. Then, sections were rinsed with running tap water for $10 \mathrm{~min}$ and covered with a coverslip using $10 \%$ glycerol in PBS.

Combined oil red $\mathrm{O}$ and immunofluorescence staining

To permit examination of lipids stained by oil red $\mathrm{O}$ together with immunolabelled cellular constituents we used the following protocol. Cryosections were fixed in $3.7 \%$ formaldehyde for $1 \mathrm{~h}$. Slides were rinsed thrice in deionised water for $30 \mathrm{~s}$ to remove excess formaldehyde. Then, sections were treated with $0.5 \%$ Triton $\mathrm{X}-100$ in PBS for $5 \mathrm{~min}$ and washed with three exchanges of PBS for $5 \mathrm{~min}$.

Sections were incubated for $30 \mathrm{~min}$ at room temperature with a primary antibody in the appropriate dilution. Here we show results from a monoclonal antibody raised against adult human slow myosin heavy chain [A4.951, developed by Dr. Blau (Cho et al. 1993)]. Incubation was followed by 15 min washing with three exchanges of PBS. Then, the appropriate fluorescein isothiocyanate (FITC)-conjugated antibody (here we used GAM-IgGFITC; SBA, Birmingham, Ala., USA) was applied for $30 \mathrm{~min}$ at room temperature. It could be argued that detergents like Triton X-100 or Tween 20 (commonly used to prevent aspecific antibody binding) may lead to loss of lipids during incubations. To check this, sections were stained with and without Triton X-100 and antibody dilutions were made in PBS with or without Tween 20.

After $15 \mathrm{~min}$ washing with three exchanges of PBS, glass slides were immersed in the working solution of oil red $\mathrm{O}$ for $30 \mathrm{~min}$. Slides were rinsed thrice with deionised water followed by 10 min with running tap water. Stained sections were embedded in $10 \%$ glycerol in $10 \mathrm{mM}$ TRIS- $\mathrm{HCl}, \mathrm{pH} 8.5$, containing DAPI to visualise nuclei and covered with a coverslip. Table 1 provides an overview of the staining procedures used. 
Table 1 Summarised staining protocol for oil red $\mathrm{O}(O R O)$ with and without immunofluorescence $(I F)$. (' Minutes, " seconds, $a b$ antibody)

${ }^{a}$ Has also been performed without Triton X-100 to test the potential delipidising effect of Triton X-100

${ }^{\mathrm{b}}$ Has also been performed with $0.1 \%$ Tween 20 in PBS in the antibody incubation media

\begin{tabular}{lll}
\hline Treatment & ORO & ORO with IF \\
\hline Fixation & $3.7 \%$ formaldehyde for $60^{\prime}$ & $3.7 \%$ formaldehyde for $60^{\prime}$ \\
Rinsing & $3 \times 30^{\prime \prime}$ with deionised water & $3 \times 30^{\prime \prime}$ with deionised water \\
Pretreatment & $-5 \%$ Triton X-100 in PBS for $5^{\prime}$ \\
Rinsing & - & $3 \times 5^{\prime}$ with PBS \\
Incubation & - & Primary ab in PBS for $30^{\prime}$ \\
Rinsing & - & $3 \times 5^{\prime}$ with PBS \\
Incubation & - & Secondary ab in PBS for $30^{\prime}$ \\
Rinsing & - & $3 \times 5^{\prime}$ with PBS \\
Staining & $30^{\prime}$ in ORO working solution & $30^{\prime}$ in ORO working solution \\
Rinsing & $3 \times 30^{\prime \prime}$ with deionised water & $3 \times 30^{\prime \prime}$ with deionised water \\
Rinsing & $10^{\prime}$ with running tap water & $10^{\prime}$ with running tap water \\
Mounting & $10 \%$ glycerol in PBS & $10 \%$ glycerol in PBS with DAPI \\
\hline
\end{tabular}

Image capturing, processing and analyses

All sections were examined using a Nikon E800 fluorescence microscope (Uvikon, Bunnik, The Netherlands) coupled to a Basler A113 C (nowadays referred to as A101 C) progressive scan colour CCD camera, with a Bayer colour filter, an output picture resolution of $1300 \times 1030$ pixels $(\mathrm{H} \times \mathrm{V})$ and a pixel size of $6.7 \times 6.7 \mu \mathrm{m}$. Oil red O-stained sections were examined in brightfield as well as in epifluorescence using a Texas red excitation filter $(540-580 \mathrm{~nm}$ ). In sections with combined dyes (oil red $\mathrm{O}$, FITC and DAPI) the following filters were used, respectively: Texas red excitation filter, FITC excitation filter $(465-495 \mathrm{~nm})$ and DAPI UV excitation filter $(340-380 \mathrm{~nm})$. Digitally captured images were processed and analysed using Lucia 5.49 software (Nikon, Düsseldorf, Germany). The amount of lipid detected in bright-field was compared with the lipid content as observed in epifluorescence in the same section. This was performed by comparing the intensity raised by the staining in an intensely labelled area with the intensity found in a more weakly labelled area. The ratio of staining intensity of these two areas was computed in bright-field as well as in epifluorescence. This procedure was performed at different areas as well as in different sections. The ratio of staining intensity obtained in bright-field was compared with the ratio of staining intensity obtained in epifluorescence by paired Student's $t$-tests. If no significant $(P<0.05)$ differences were found, this implies that examination of the same section in bright-

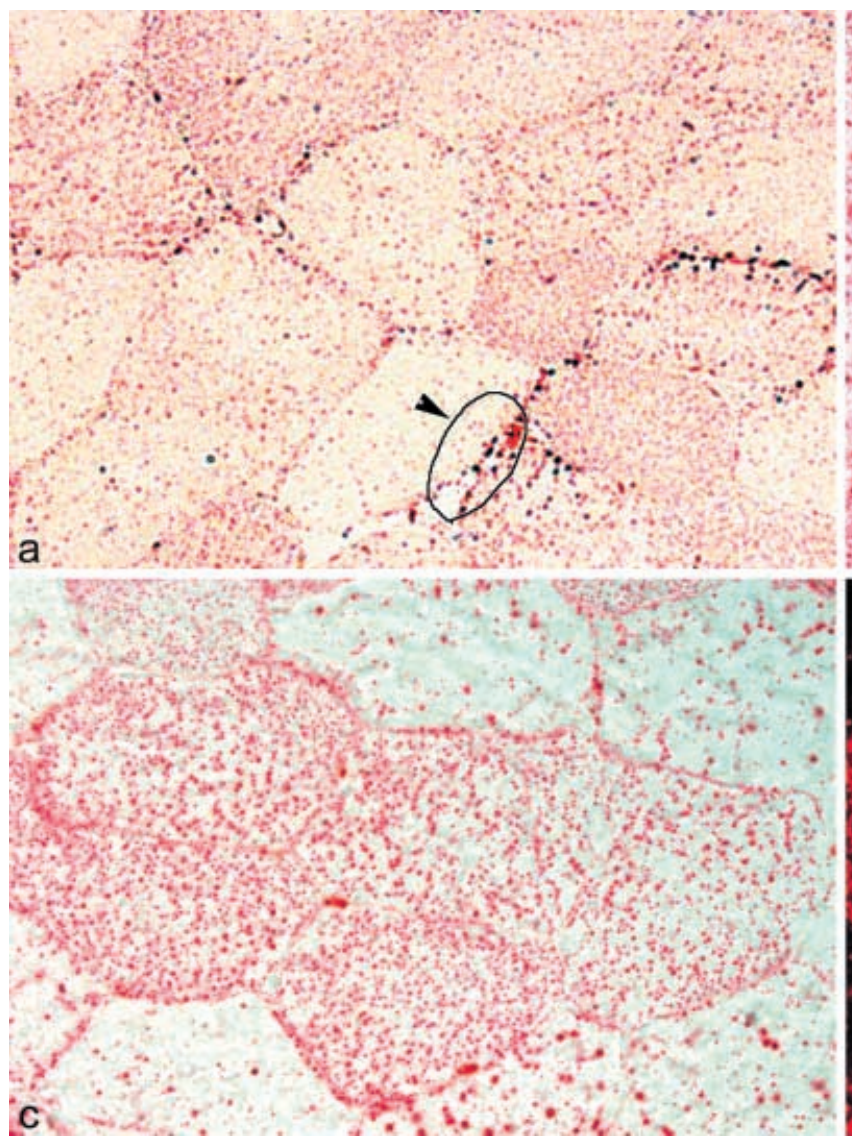

Fig. 1 a Results of oil red $\mathrm{O}$ staining according to Goodpaster (2000b). b Modified procedure described here. Encircled, and indicated by arrowhead in a, crystallised oil red $\mathrm{O}$ can be observed. In $\mathbf{b}$, the single arrowhead shows the lumen of a blood vessel

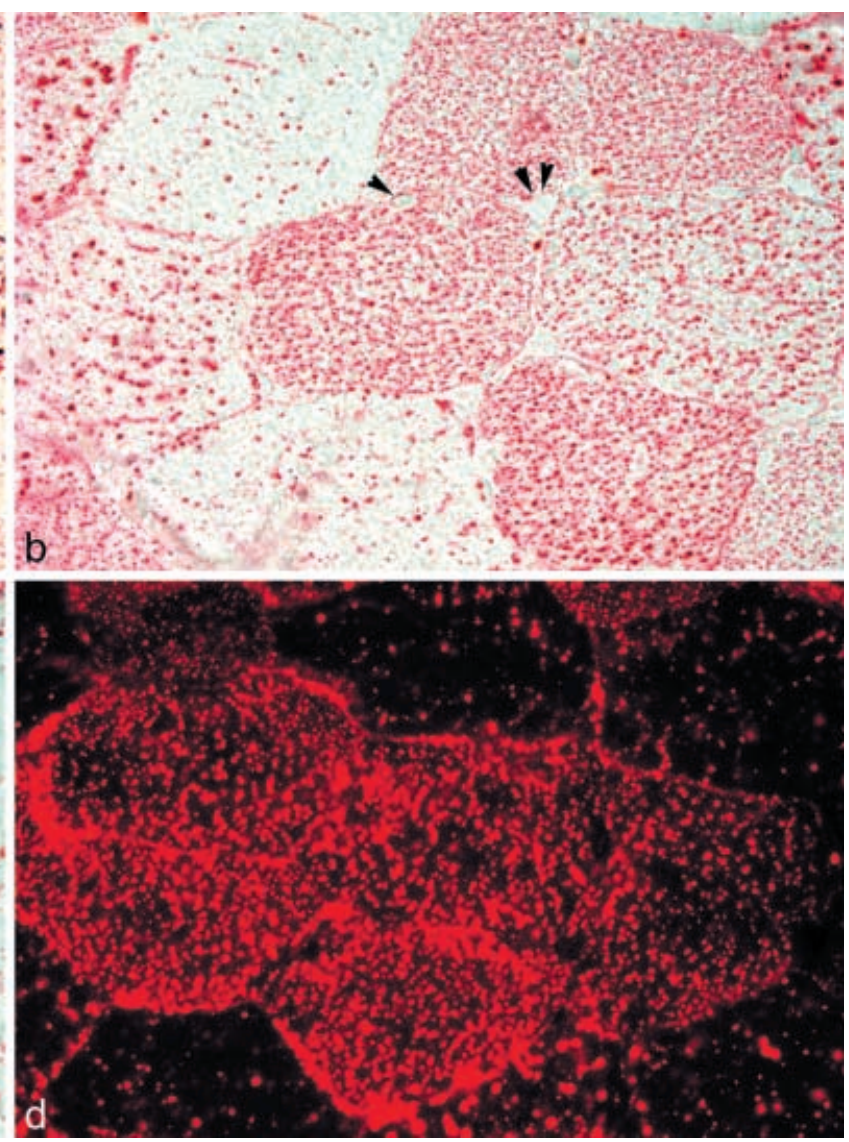

which lacks oil red $\mathrm{O}$ staining, while the double arrowheads indicate extramyocellular space, lacking oil red O staining. c, d Same section examined in bright-field (c) or in epifluorescence (d) 

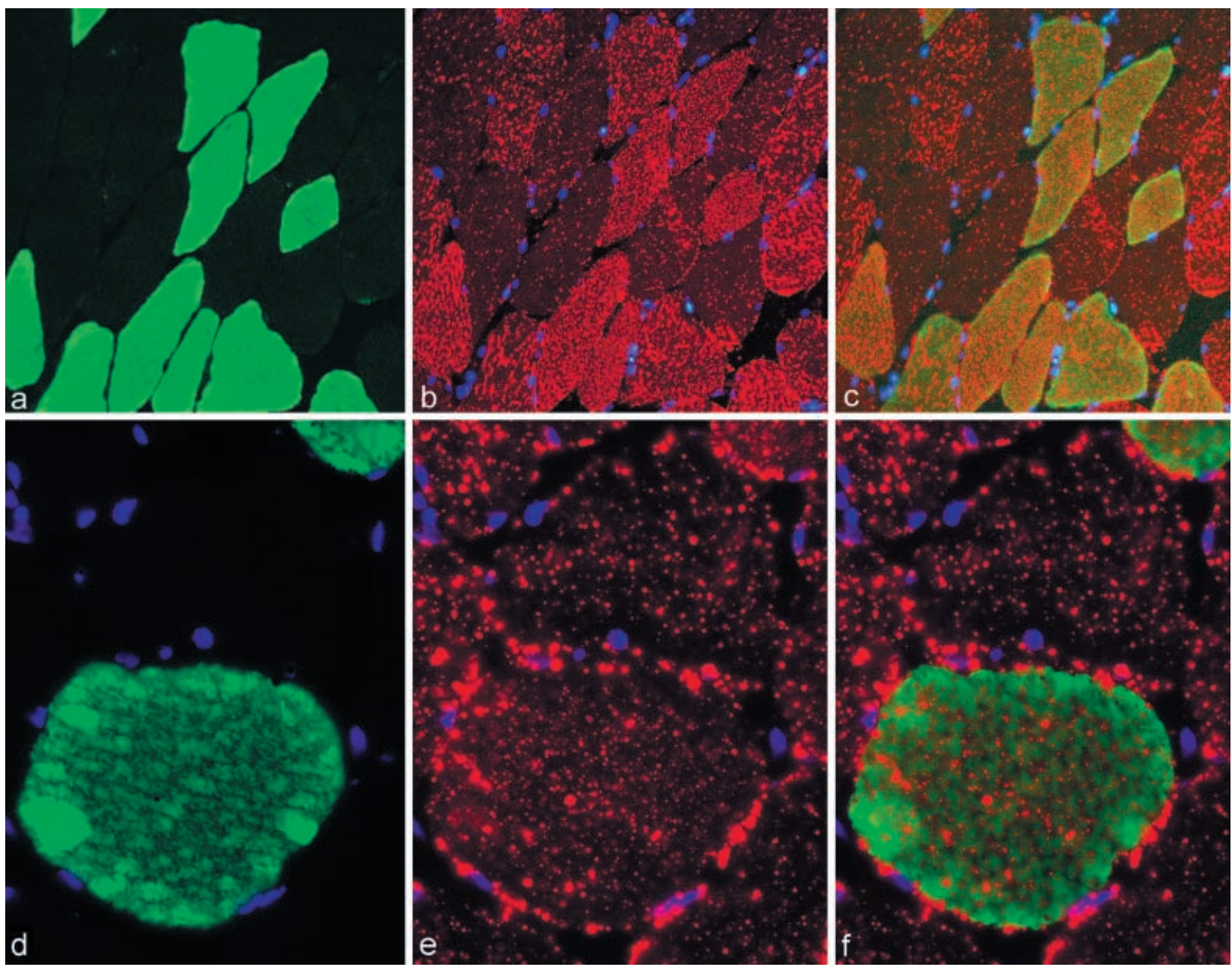

Fig. 2 a, d Immunofluorescence against slow myosin heavy chain (sMHC). Oil red $\mathrm{O}$ staining is shown in $\mathbf{b}$ and $\mathbf{c}$, while an overlay of both stains is shown in $\mathbf{c}$ and $\mathbf{f}$. Please note that the fibres identified as slow oxidative fibres by staining for sMHC also possess an abundant lipid deposition

field or epifluorescence yields similar results with regard to the lipid content.

\section{Results}

The protocols presented here were equally successful in human and rat muscles. All results shown here originate from human vastus lateralis muscle. Treatment of serial sections with Triton X-100, or adding Tween 20 to the antibody incubation media, did not affect the lipid content (data not shown). All results presented in epifluorescence are derived from Triton X-100-treated sections. Antibodies were diluted in PBS without Tween 20 in sections shown in Fig. $2 \mathrm{a}-\mathrm{c}$ and with Tween 20 in Fig. 2d-f.

To prevent background labelling, the original protocol (Lillie and Ashburn 1943) includes differentiation with isopropanol, which in our hands completely abolished the oil red $\mathrm{O}$ signal (results not shown). Results from staining a human section according to Goodpaster (2000b) are shown in Fig. 1a. As indicated by arrowheads in Fig. 1a, some oil red O crystals are still present, despite filtration of the oil red $\mathrm{O}$ working solution. Intramyocellular lipids are clearly labelled by the oil red $\mathrm{O}$ dye and intercellular differences in lipid content can be observed. Figure $1 \mathrm{~b}$ shows an oil red O-stained section following the modified protocol described here (i.e. after formaldehyde fixation and dilution of oil red $\mathrm{O}$ in $36 \%$ triethyl phosphate instead of $60 \%$ isopropanol). Again, clear intercellular differences in staining intensity are detectable. However, the signal raised using the modified protocol lacks oil red $\mathrm{O}$ crystals, is more punctate and neither the lumen of small blood vessels (indicated by arrowheads in Fig. 1b) nor the extramyocellular space (indicated by a double arrowhead in Fig. 1b) are stained with oil red $\mathrm{O}$.

Figure 1c shows a representative section of human vastus lateralis using the optimised oil red $\mathrm{O}$ staining examined in bright-field microscopy, while Fig. 1d shows the same section and exactly the same field examined in epifluorescence microscopy. Images were successively captured in bright-field and fluorescence microscopy. As oil red $\mathrm{O}$ can be excited at wavelengths between 540 and $580 \mathrm{~nm}$, the Texas red filter can be used to examine the signal in fluorescence microscopy. After signal quantification using Lucia 4.59 software, we calculated the 
Table 2 The computed staining intensity ratio of nine (randomly chosen) weakly and intensely stained areas shown for bright-field $(B F)$ and epifluorescence $(E F)$. Mean intensity in $\mathrm{BF}$ did not differ significantly from $\mathrm{EF}(P>0.05)$ indicating that examination of lipid deposition by oil red $\mathrm{O}$ can be performed in BF or EF with similar results

\begin{tabular}{lll}
\hline Area & Ratio BF & Ratio EF \\
\hline 1 & 0.95 & 0.83 \\
2 & 0.34 & 0.22 \\
3 & 1.82 & 2.31 \\
4 & 1.22 & 0.99 \\
5 & 1.50 & 1.43 \\
6 & 1.46 & 1.27 \\
7 & 0.86 & 1.34 \\
8 & 1.23 & 1.45 \\
9 & 0.48 & 0.14 \\
Mean & 1.17 & 1.23 \\
SD & 0.48 & 0.67 \\
\hline
\end{tabular}

staining intensity ratio of two different areas randomly chosen at various sites on a section. This procedure was performed in bright-field and epifluorescence. Table 2 shows the results of a random selection of areas. No significant differences could be detected between the ratios computed in bright-field compared to fluorescence.

Oil red $\mathrm{O}$ combined with immunofluorescence assays is shown in Fig. 2. Figure 2a shows muscle fibres expressing slow myosin heavy chain (also referred to as type 1 or slow oxidative fibres) adjacent to fibres of a distinct fibre type, judged by the lack of slow myosin expression. Lipid localisation by oil red $\mathrm{O}$ is shown in Fig. 2b, indicating fibres with a low and a high abundance of myocellular lipids. An overlay of the immunolabelling with oil red $\mathrm{O}$ is shown in Fig. 2c. Obviously, the fibres expressing slow myosin heavy chain correspond to the fibres with a more abundant lipid signal, which was to be expected considering the high capacity of slow (type 1) fibres to oxidise lipids. In Fig. 2b, c, nuclei are stained blue by DAPI. A higher magnification of a different section is shown in Fig. 2d-f, using $0.1 \%$ Tween 20 in the incubation steps.

\section{Discussion}

In order to perform computerised quantification of lipids in muscle sections while concurrently examining other, immunolabelled, subcellular constituents, we aimed to optimise oil red $\mathrm{O}$ for use in quantitative fluorescence microscopy.

Neutral lipids can be examined histologically by different stains, all of which use lipid-soluble dyes as a marker. Candidate dyes to study lipid deposition are Sudan black or Nile blue. Of these dyes Sudan black is considered to be the most sensitive (Hopkins et al. 1983). However, the dark blue to black stain yielded restricts its application to single-staining techniques. Alternatively, Nile blue could have been used as a dye. However, Nile blue stains neutral lipids, phospholipids and free fatty acids. As mainly neutral lipid droplets and not free fatty acids are of interest in studies linking IMTG to insulin resistance, Nile blue (staining) is not compatible with our purposes. Application of lipid stains for fluorescence microscopy has been performed in the mid-eighties by using Nile red as a dye in cells (Greenspan et al.
1985) as well as in tissue sections (Fowler and Greenspan 1985). The disadvantage of using Nile red for our application is that in addition to neutral lipids also phospholipids, cholesterol, cholesteryl esters and free albumin possess fluorescence upon Nile red staining (Greenspan and Fowler 1985). Considering the limitations of the above-mentioned dyes, we have chosen to use oil red $\mathrm{O}$ in the present study.

The main finding of the present study is the development of a staining protocol which permits quantitative fluorescence microscopy of both oil red O-stained lipids and other (immuno)labelled structures. Before this goal was achieved, we encountered some drawbacks of the conventional staining method. Crystal formation was prevented by diluting oil red $\mathrm{O}$ in $36 \%$ triethyl phosphate. Retention of the lipids within the sections was improved by formaldehyde fixation while background staining was successfully abolished by differentiation with $36 \%$ triethyl phosphate. The use of detergents like Triton X-100 or Tween 20 did not induce delipidisation of the sections. Following this optimisation we can conclude that quantification of oil red O-stained lipid droplets in bright-field or in epifluorescence microscopy yields similar results.

The observation that oil red $\mathrm{O}$ can be examined in epifluorescence microscopy using a 540- to 580-nm Texas red excitation/emission filter has several advantages above conventional bright-field examination. First, it is known that phospholipids of some, but not all, membranes are stained pink by oil red O (Bayliss-High 1977). Upon conversion from colour to greyscale the pink, phospholipid-derived signal interferes with the red signal from the lipid droplets. Therefore, under conditions when phospholipids are significantly labelled, conversion of colour images to greyscale may induce overestimation of the lipid droplet content. The pink signal will be lost from the excitation spectrum using a Texas red filter and can therefore no longer interfere with the lipid droplet derived signal. Thus, examination of oil red $\mathrm{O}$ in epifluorescence will solely identify lipid droplets and not phospholipids. Second, double and even multi-immunofluorescence stains can be performed on one single section.

Also, we serendipitously observed that, despite fixation with formaldehyde and storage at a cool dark place, the oil red $\mathrm{O}$ signal was almost completely abolished within 2 weeks, but not within 3 days, after staining.

In conclusion, the present study describes a protocol for computerised quantification of oil red O-stained lipid droplets in skeletal muscle sections. The protocol presented here permits examination of lipid droplets stained by oil red O together with multiple staining of all other immunodetectable myocellular constituents by quantitative immunofluorescence within the same muscle section.

Acknowledgements The antibody against adult human myosin heavy chain (referred to as A4.951) used in the present study was developed by Dr. Blau and was obtained from the Developmental Studies Hybridoma Bank, developed under the auspices of the NICHD and maintained by The University if Iowa, Department of Biological Sciences, Iowa City, IA 52242, USA. 


\section{References}

Bayliss-High O (1977) Lipids. In: Bancroft JD, Stevens A (eds) Theory and practice of histological techniques. Churchill Livingstone, Edinburgh, pp 168-185

Boesch C, Slotboom J, Hoppeler H, Kreis R (1997) In vivo determination of intra-myocellular lipids in human muscle by means of localized 1H-MR-spectroscopy. Magn Reson Med 37:484-493

Cho M, Webster SG, Blau HM (1993) Evidence for myoblastextrinsic regulation of slow myosin heavy chain expression during muscle fiber formation in embryonic development. J Cell Biol 121:795-810

Dyck DJ, Bonen A (1998) Muscle contraction increases palmitate esterification and oxidation and triacylglycerol oxidation. Am J Physiol 275:E888-E896

Dyck DJ, Peters SJ, Glatz J, Gorski J, Keizer H, Kiens B, Liu S, et al (1997) Functional differences in lipid metabolism in resting skeletal muscle of various fiber types. Am J Physiol 272: E340-E351

Fowler SD, Greenspan P (1985) Application of Nile red, a fluorescent hydrophobic probe, for the detection of neutral lipid deposits in tissue sections: comparison with oil red O. J Histochem Cytochem 33:833-836

Goodpaster BH, Kelley DE, Thaete FL, He J, Ross R (2000a) Skeletal muscle attenuation determined by computed tomography is associated with skeletal muscle lipid content. J Appl Physiol 89:104-110

Goodpaster BH, Theriault R, Watkins SC, Kelley DE (2000b) Intramuscular lipid content is increased in obesity and decreased by weight loss. Metabolism 49:467-472

Greenspan P, Fowler SD (1985) Spectrofluorometric studies of the lipid probe, Nile red. J Lipid Res 26:781-789

Greenspan P, Mayer EP, Fowler SD (1985) Nile red: a selective fluorescent stain for intracellular lipid droplets. J Cell Biol 100:965-973
Guo Z, Jensen MD (1998) Intramuscular fatty acid metabolism evaluated with stable isotopic tracers. J Appl Physiol 84: 1674-1679

Hopkins D, Manchester KL, Gregory M (1983) Histochemical and biochemical characteristics of the transient hypertrophy of the denervated rat hemidiaphragm. Exp Neurol 81:279-293

Krssak M, Falk Petersen K, Dresner A, DiPietro L, Vogel SM, Rothman DL, Roden M, et al (1999) Intramyocellular lipid concentrations are correlated with insulin sensitivity in humans: a 1H NMR spectroscopy study. Diabetologia 42: $113-116$

Lillie RD, Ashburn LL (1943) Supersaturated solutions of fat stains in dilute isopropanol for demonstration of acute fatty degeneration not shown by Herxheimer's technique. Arch Pathol 36:432-440

Pan DA, Lillioja S, Kriketos AD, Milner MR, Baur LA, Bogardus C, Jenkins AB, et al (1997) Skeletal muscle triglyceride levels are inversely related to insulin action. Diabetes 46:983-988

Pette D, Peuker H, Staron RS (1999) The impact of biochemical methods for single muscle fibre analysis. Acta Physiol Scand 166:261-277

Russell JC, Shillabeer G, Bar-Tana J, Lau DC, Richardson M, Wenzel LM, Graham SE, et al (1998) Development of insulin resistance in the JCR:LA-cp rat: role of triacylglycerols and effects of MEDICA 16. Diabetes 47:770-778

Schrauwen P, Wagenmakers AJ, Marken Lichtenbelt WD van, Saris WH, Westerterp KR (2000) Increase in fat oxidation on a high-fat diet is accompanied by an increase in triglyceridederived fatty acid oxidation. Diabetes 49:640-646

Vusse vd GJ, Reneman RS (1996) Lipid metabolism in muscle. In Rowell LB, Shepherd JT (eds) Handbook of physiology, section 12. Exercise: regulation and integration of multiple systems. Oxford University Press, New York, pp 953-994 